\title{
Abandono de psicoterapia com crianças
}

\author{
Child psychotherapy dropout
}

\author{
Elisabeth Kuhn Deakin'1, Maria Lucia Tiellet Nunes² \\ ${ }^{1}$ Doutora, Psicologia, Pontifícia Universidade Católica do Rio Grande do Sul (PUCRS), Porto Alegre, RS. ${ }^{2}$ Doutora, Psicologia, Universidade de Berlim, Berlim, Alemanha. \\ Coordenadora, Programa de Pós-Graduação em Psicologia, PUCRS. \\ Este estudo foi realizado no Programa de Pós-Graduação em Psicologia, Pontifícia Universidade Católica do Rio Grande do Sul (PUCRS), Porto Alegre, RS.
}

\section{Resumo}

Objetivo: Comparar uma amostra de 24 crianças que completaram 12 meses de psicoterapia psicanalítica com uma amostra de 38 crianças que interromperam prematuramente o tratamento.

Método: Trata-se de um estudo quantitativo em dois grupos de crianças; tratamento quase-experimental de medida única antes do início da psicoterapia psicanalítica individual.

Resultados: Foram encontradas diferenças estatisticamente significantes nas seguintes variáveis: sexo, sintomas, frequência dos atendimentos, capacidade da criança de controlar as emoções e os impulsos, medida pelo teste Rorschach e nas escalas de sociabilidade, queixas somáticas e comportamentos internalizantes, tais como ansiedade e depressão, medidas pelo Child Behavior Checklist.

Conclusões: Este estudo revelou que a psicoterapia psicanalítica é efetiva no tratamento da criança do sexo feminino que apresenta transtornos internalizantes, tais como ansiedade e depressão, tem maior descontrole de emoções e impulsos e recebe atendimento com uma frequência de duas vezes por semana por pelo menos 12 meses.

Descritores: Psicoterapia psicanalítica com crianças, pesquisa de resultados, psicanálise, efetividade e abandono.

\begin{abstract}
Objective: To compare a sample of 24 children who completed 12 months of psychoanalytical psychotherapy with a sample of 38 children who dropped out from treatment before 12 months of intervention.

Method: Quantitative study involving two groups of children. Quasi-experimental treatment of single measure before the beginning of individual psychoanalytical psychotherapy.

Results: Statistically significant differences were found in the following variables: gender, symptoms, frequency of sessions, child's impulse and emotion control (measured using the Rorschach test and sociability scales), somatic complaints and internalizing behaviors, such as anxiety and depression (measured using the Child Behavior Checklist).

Conclusions: The results revealed that child psychoanalytical psychotherapy is effective in the treatment of female children with internalizing disorders, such as anxiety and depression, who have little control of impulses and emotions and attend at least 12 months of psychoanalytical psychotherapy twice a week.

Keywords: Child psychoanalytical psychotherapy, outcome research, psychoanalysis, effectiveness and dropout.

\section{Introdução}

O abandono do tratamento psicoterápico é um problema de grande relevância para aqueles que oferecem serviços a crianças, adolescentes e suas famílias. Estudos longitudinais indicam que as doenças psiquiátricas na infância são precursoras de uma gama de resultados negativos, ou seja, se uma

criança sofre de uma doença psiquiátrica na infância e não é tratada, ela tem maior risco de desenvolver outros transtornos emocionais ao longo de sua vida adulta ${ }^{1}$. Neste sentido, é fundamental a compreensão empírica do fenômeno que envolve a interrupção prematura do tratamento psicoterápico de crianças para que ações preventivas apropriadas possam ser tomadas, e, assim, mais crianças possam vir a ser atendidas.
\end{abstract}

\section{Correspondência:}

Maria Lucia Tiellet Nunes, Av. Ipiranga, 6681, Prédio 11, $9^{\circ}$ andar, CEP 90619 -900, Porto Alegre, RS. E-mail: elisabethkdeakin@hotmail.com

Não foram declarados conflitos de interesse associados à publicação deste artigo.

Copyright (C) Revista de Psiquiatria do Rio Grande do Sul - APRS

Recebido em 09/10/2008. Aceito em 09/03/2009. 
Para ir ao encontro desta demanda, foi desenvolvido este estudo, que teve como objetivo comparar um grupo de 62 crianças que chegaram a atendimento psicológico por problemas emocionais diversos em um ambulatório de Porto Alegre (RS). Destas 62 crianças, uma recebeu alta após 7 meses de atendimento, e 23 completaram 12 meses de psicoterapia psicanalítica. As demais 38 crianças interromperam o tratamento, denotando um índice de abandono prematuro de $61,3 \%$.

\section{Abandono prematuro do tratamento}

Neste estudo, considerou-se abandono prematuro de tratamento a interrupção da psicoterapia psicanalítica antes de completar 12 meses de tratamento por decisão unilateral do paciente e/ou sua família, sem a concordância do terapeuta, que considerava o tratamento incompleto.

Existem muitos fatores citados na literatura relacionados ao abandono prematuro. São estes: a) percepção por parte da criança e/ou família de que o tratamento não é relevante; b) uma aliança terapêutica frágil com a criança e sua família no início do tratamento; c) desvantagem socioeconômica; d) alto nível de estresse e disfunção na família, entre outros. Na verdade, quanto mais fatores presentes, maior o risco de abandono prematuro. Os estudos indicam que, na maioria dos casos, a razão do abandono reside nas dificuldades familiares e não na criança que necessita atendimento ${ }^{2-4}$.

Neste sentido, a psicoterapia com crianças difere da psicoterapia com adultos, já que a criança não busca tratamento para si mesma e, portanto, depende da motivação dos pais para que se mantenha em tratamento. Por esse motivo, uma aliança terapêutica positiva com os pais é, muitas vezes, mais importante para a manutenção do tratamento do que uma boa aliança terapêutica com a criança ${ }^{5}$. Na maioria dos casos, os pais participam ativamente da psicoterapia de seus filhos. Quanto maior a resistência para a participação, maior o risco de abandono prematuro ${ }^{3,5}$.

\section{Objetivos}

Comparar o grupo de crianças que se manteve em psicoterapia psicanalítica por 12 meses e que seguiu o tratamento até o momento da alta combinada (G1) com o grupo de crianças que abandonou prematuramente o tratamento (G2). Também, procurou-se realizar uma associação entre as variáveis sociodemográficas, clínicas e dos resultados dos testes com a permanência em psicoterapia psicanalítica.

\section{Método}

Trata-se de um estudo quantitativo em dois grupos (G1 versus $\mathrm{G} 2$ ); tratamento quase-experimental de medida única antes do início da psicoterapia psicanalítica individual. Os dados sociodemográficos, clínicos e dos resultados dos testes foram associados com a permanência na psicoterapia psicanalítica por 12 meses e seguimento do tratamento até a alta combinada e com o abandono da psicoterapia antes dos 12 meses ou por alta combinada.

\section{Amostra}

Composta de 62 crianças que buscaram atendimento psicoterápico no Centro de Estudos, Atendimento e Pesquisa da Infần cia e Adolescência (CEAPIA), em Porto Alegre (RS), nos anos de 2005 e 2006, e concordaram em participar da pesquisa. A amostra foi, então, dividida em dois grupos: o G1, que corresponde às crianças que se mantiveram em atendimento por 12 meses e seguiram em atendimento e/ou tiveram alta combinada, perfazendo um total de 24 crianças, e o G2, que corresponde às crianças que abandonaram o tratamento, com um total de 38 crianças. As características sociodemográficas da amostra são apresentadas na Tabela 1 .

Tabela 1 - Características sociodemográficas

\begin{tabular}{lccc}
\hline Características & G1, n (\%) & G2, n (\%) & Total, n (\%) \\
\hline Sexo & & & \\
$\quad$ Feminino & $15(62,5)$ & $8(21,1)$ & $23(37,1)$ \\
$\quad$ Masculino & $9(37,5)$ & $30(78,9)$ & $39(62,9)$ \\
Idade & & \\
6 anos & $5(20,8)$ & $8(21,1)$ & $13(21,0)$ \\
7 anos & $3(12,5)$ & $5(13,2)$ & $10(12,9)$ \\
8 anos & $4(16,7)$ & $8(21,1)$ & $12(19,4)$ \\
9 anos & $6(25,0)$ & $9(23,7)$ & $15(24,2)$ \\
10 anos & $4(16,7)$ & $5(13,2)$ & $9(14,5)$ \\
11 anos & $2(8,3)$ & $3(7,9)$ & $5(8,1)$ \\
Escolaridade & & $4(6,5)$ \\
Pré-escola & $2(8,3)$ & $2(5,3)$ & $13(21,0)$ \\
Primeira série & $4(16,7)$ & $9(23,7)$ & $11(17,7)$ \\
Segunda série & $2(8,3)$ & $9(23,7)$ & $15(24,2)$ \\
Terceira série & $7(29,2)$ & $8(21,1)$ & $11(17,7)$ \\
Quarta série & $5(20,8)$ & $6(15,8)$ & $7(11,3)$ \\
Quinta série & $3(12,5)$ & $4(10,5)$ & \\
\hline
\end{tabular}

G1 = grupo que se manteve em psicoterapia até a alta combinada; $\mathrm{G} 2$ = grupo que abandonou a psicoterapia antes da alta combinada. 
A análise das frequências indica que no momento da busca a amostra era constituída de $37,1 \%$ de crianças do sexo feminino e $62,9 \%$ de crianças do sexo masculino. Apesar de ser a minoria no momento inicial deste estudo, as crianças do sexo feminino são a maioria $(62,5 \%)$ no grupo que permaneceu em psicoterapia durante 12 meses e não interrompeu o tratamento. As crianças do sexo masculino, por sua vez, formaram a maioria no grupo que abandonou prematuramente o tratamento $(78,9 \%)$.
Em relação à idade, o maior número de crianças em ambos os grupos se concentra na faixa de 8 a 10 anos. A escolaridade das crianças reflete a idade destas, já que o número de repetências é praticamente nulo.

As características clínicas da amostra são apresentadas na Tabela 2.

Os resultados indicam que a escola foi a fonte de maior encaminha mento em ambos os grupos, seguida por profissionais

Tabela 2 - Características clínicas

\begin{tabular}{|c|c|c|}
\hline Características & G1, n (\%) & G2, n (\%) \\
\hline \multicolumn{3}{|l|}{ Encaminhamento } \\
\hline Escola & $10(41,7)$ & $13(34,2)$ \\
\hline Neurologista & $2(8,3)$ & $2(5,3)$ \\
\hline Psiquiatra & $3(12,5)$ & $3(7,9)$ \\
\hline Pediatra & $2(8,3)$ & $3(7,9)$ \\
\hline Psicólogo & $1(4,2)$ & $1(2,9)$ \\
\hline Familiar & $4(16,3)$ & $4(10,5)$ \\
\hline Outro & $2(8,3)$ & $9(23,7)$ \\
\hline Não tem informação & $0(0,0)$ & $3(7,9)$ \\
\hline \multicolumn{3}{|l|}{ Motivos de busca } \\
\hline Problemas escolares & $7(29,2)$ & $7(18,4)$ \\
\hline Dificuldades nas relações interpessoais & $5(20,8)$ & $5(13,2)$ \\
\hline Falta de atenção & $3(12,5)$ & $12(31,6)$ \\
\hline Depressão e tristeza & $8(33,3)$ & $3(7,9)$ \\
\hline Dificuldade no sono/alimentação/ controle dos esfíncteres & $1(4,2)$ & $1(2,6)$ \\
\hline Problemas de comportamento & $5(20,8)$ & $15(39,5)$ \\
\hline Ansiedade/insegurança/medos & $17(70,8)$ & $13(29,2)$ \\
\hline Agressividade & $7(29,2)$ & $7(18,4)$ \\
\hline \multicolumn{3}{|l|}{ Hipótese diagnóstica inicial } \\
\hline Transtorno de humor & $4(16,7)$ & $10(26,3)$ \\
\hline Transtorno de conduta & $9(29,2)$ & $15(10,5)$ \\
\hline Transtorno de ansiedade & $7(37,5)$ & $4(39,5)$ \\
\hline TDAH & $2(8,3)$ & $4(10,5)$ \\
\hline Outros & $2(8,3)$ & $4(10,5)$ \\
\hline Não tem informação & $0(0,0)$ & $1(206)$ \\
\hline \multicolumn{3}{|l|}{ Número de sessões por semana } \\
\hline Uma sessão por semana & $11(45,8)$ & $19(50,0)$ \\
\hline Duas sessões por semana & $13(54,2)$ & $3(7,9)$ \\
\hline Só avaliação psicológica & $0(0,0)$ & $14(36,8)$ \\
\hline Não tem informação & $0(0,0)$ & $2(5,3)$ \\
\hline \multicolumn{3}{|l|}{ Tratamentos concomitantes } \\
\hline Sim & $8(33,3)$ & $5(13,2)$ \\
\hline Não & $16(66,7)$ & $33(86,8)$ \\
\hline \multicolumn{3}{|l|}{ Uso de medicação } \\
\hline $\operatorname{Sim}$ & $6(25,0)$ & $3(7,9)$ \\
\hline Não & $18(75,0)$ & $35(92,1)$ \\
\hline \multicolumn{3}{|l|}{ Ano do curso do terapeuta } \\
\hline Estágio de psicologia & $2(8,3)$ & $3(7,9)$ \\
\hline Primeiro ano & $5(20,8)$ & $17(44,7)$ \\
\hline Segundo ano & $14(58,3)$ & $10(26,3)$ \\
\hline Terceiro ano/corpo clínico & $3(12,5)$ & $8(15,8)$ \\
\hline
\end{tabular}

$\overline{\mathrm{G} 1}$ = grupo que se manteve em psicoterapia até a alta combinada; $\mathrm{G} 2$ = grupo que abandonou a psicoterapia antes da alta combinada; TDAH = transtorno do déficit de atenção com hiperatividade. 
da saúde. Em relação aos motivos de busca, os comportamentos internalizantes, como ansiedade (70,8\%) e depressão $(33,3 \%)$ predominam no G1, mostrando-se presentes em um grande número de casos. Por outro lado, os comportamentos externalizantes, como problemas de comportamento, são a maioria no G2, presentes em $39,5 \%$ dos casos.

Como hipótese diagnóstica inicial, a maioria dos casos em ambos os grupos se concentra nos transtornos ligados à ansiedade, o que pode ser relacionado aos motivos de busca no G1, mas não aos motivos de busca do G2. A maioria dos pacientes foi atendida duas vezes por semana no G1 $(54,8 \%)$. Já no G2, a maior parte dos casos só realizou a avaliação psicológica $(36,8 \%)$, ou foi atendida uma vez por semana $(47,4 \%)$. A avaliação psicológica neste estudo consistia em aproximadamente duas sessões com os pais, duas sessões com a criança e uma sessão de devolução com os pais, na qual o plano de tratamento era discutido, totalizando cinco sessões. Os resultados também indicam que $86,8 \%$ das crianças do G2 não realizavam outros atendimentos concomitantes à psicoterapia. No G1, este número corresponde a $66,7 \%$. Quanto ao ano do curso no qual o terapeuta se encontrava no momento da busca, $20,8 \%$ dos terapeutas do G1 estavam no primeiro ano e $58,3 \%$, no segundo ano. Já no G2, apenas $26,3 \%$ estavam no segundo ano, sendo que a grande maioria dos terapeutas estava concentrada no primeiro ano do curso $(44,7 \%)$. O curso oferecido pelo serviço é de formação em psicoterapia da infância e adolescência e tem duração de 3 anos.

\section{Instrumentos}

Para a avaliação das 62 crianças foram utilizados os seguintes instrumentos: a) ficha de levantamento sociodemográfico e clínico das crianças avaliadas; b) teste Gestáltico Visomotor de Bender ${ }^{6}$; c) The Wechsler Intelligence Scale for Children (WISC III) com padronização brasileira de Figueiredo ${ }^{7}$; d) técnica de Rorschach; e d) Child Behavior Checklist (CBCL).

\section{Procedimento de análise dos dados}

No teste Bender Gestalt, duas escalas para pontuação de escores foram utilizadas: a escala de maturação segundo a idade conforme Koppitz ${ }^{8}$ e a escala de maturação segundo a idade conforme Kroeff 9 . No WISC III, a escala utilizada para avaliação dos resultados foi a adaptação e padronização desse instrumento a partir de uma amostra brasileira de Vera Lúcia Marques de Figueiredo ${ }^{7}$. Já no Rorschach, os resultados foram levantados e interpretados a partir do sistema compreensivo para crianças proposto por Exner'. E, por fim, o CBCL foi levantado a partir de um programa de computador que apresenta os escores e os divide em clínico, limítrofe e não clínico ${ }^{10}$.

Em relação à ficha preenchida pelos terapeutas, o levantamento foi feito a partir das informações fornecidas por estes quanto aos dados sociodemográficos e clínicos.
Os resultados de cada teste e das fichas de cada criança foram transportados para o programa Statistical Package for Social Sciences (SPSS) 11.0. Foi, então, aplicado o teste quiquadrado para heterogeneidade com correção de Yates, ou o teste exato de Fisher, quando necessário, para associação das variáveis categóricas. $\mathrm{O}$ teste $t$ de Student foi utilizado para a associação das variáveis quantitativas. Objetivou-se, com isto, realizar uma associação entre as variáveis sociodemográficas, clínicas e dos resultados dos testes com a permanência em psicoterapia psicanalítica por 12 meses e com o abandono do tratamento.

\section{Resultados}

Para a análise das diferenças das variáveis sociodemográficas e clínicas foi aplicado o teste qui-quadrado. Os resultados estão dispostos na Tabela 3.

Tabela 3 - Variáveis sociodemográficas e clínicas

\begin{tabular}{lc}
\hline Variáveis & p \\
\hline Idade & 0,998 \\
Sexo & 0,001 \\
Escolaridade & 0,575 \\
Encaminhamento & \\
$\quad$ Problemas escolares & 0,470 \\
$\quad$ Agressividade & 0,324 \\
$\quad$ Ansiedade/insegurança e medos & 0,005 \\
$\quad$ Depressão/tristeza & 0,011 \\
$\quad$ Problemas de relacionamento interpessoal & 0,423 \\
$\quad$ Problemas de atenção & 0,088 \\
$\quad$ Problemas com sono/alimentação/esfíncteres & 0,739 \\
$\quad$ Problemas de comportamento & 0,003 \\
$\quad$ Outros & 0,487 \\
Hipótese diagnóstica & 0,510 \\
Ano do curso do terapeuta & 0,083 \\
Frequência dos atendimentos & 0,001 \\
Atendimentos concomitantes & 0,057 \\
Uso de medicação & 0,063 \\
\hline
\end{tabular}

A análise das variáveis sociodemográficas e clínicas demonstra que houve diferenças estatisticamente significantes nas seguintes variáveis: sexo $(0,001)$, com predominância do sexo feminino no grupo que permaneceu em tratamento por 12 meses; motivos de busca: ansiedade, insegurança, medos $(0,005)$ e depressão $(0,001)$, todos presentes em maior número no $\mathrm{G} 1$, e problemas de comportamento $(0,003)$, mais presentes no G2; frequência dos atendimentos, predominando a frequência de duas vezes por semana no G1 e de uma vez por semana no G2. Todas as demais variáveis não apresentaram diferenças estatisticamente significantes. 
Em relação às variáveis relativas aos resultados dos testes Bender, WISC III, Rorschach e CBCL, o valor do p está demonstrado na Tabela 4.

Tabela 4 - Resultados dos testes Bender, WISC III, Rorschach e CBCL

\begin{tabular}{|c|c|}
\hline & $\mathbf{p}$ \\
\hline \multicolumn{2}{|l|}{ Bender Gestalt } \\
\hline Bender Koppitz & 0,577 \\
\hline \multicolumn{2}{|l|}{ Bender Gestalt } \\
\hline \multicolumn{2}{|l|}{ WISC III } \\
\hline QI verbal & 0,719 \\
\hline QI de execução & 0,719 \\
\hline QI total & 0,906 \\
\hline \multicolumn{2}{|l|}{ Rorschach } \\
\hline Número de respostas & 0,678 \\
\hline$\%$ Global & 0,825 \\
\hline$\%$ Detalhe comum & 0,795 \\
\hline$\%$ Detalhe incomum & 0,668 \\
\hline$\% \mathrm{~S}$ & 0,846 \\
\hline \% Respostas F & 0,249 \\
\hline$\%$ Fo & 0,372 \\
\hline$\% \mathrm{~F}-$ & 0,693 \\
\hline$\% \mathrm{FM}$ & 0,463 \\
\hline Numero de respostas & 0,678 \\
\hline$\%$ Global & 0,825 \\
\hline$\%$ Detalhe comum & 0,795 \\
\hline$\% \mathrm{M}$ & 0,135 \\
\hline$\% \mathrm{FC}$ & 0,197 \\
\hline$\% \mathrm{CF}$ & 0,041 \\
\hline$\% \mathrm{C}$ & 0,433 \\
\hline$\% \mathrm{FC}^{\prime}$ & 0,760 \\
\hline$\% \mathrm{C}^{\prime} \mathrm{F}+\mathrm{C}^{\prime}$ & 0,615 \\
\hline$\% \mathrm{Fm}$ & 0,135 \\
\hline$\% \mathrm{~m}+\mathrm{mF}$ & 0,013 \\
\hline$\%$ FT & 0,905 \\
\hline$\% \mathrm{~A}+\mathrm{Ad}$ & 0,174 \\
\hline$\% \mathrm{H}$ & 0,868 \\
\hline \multicolumn{2}{|l|}{ CBCL } \\
\hline Atividade & 0,890 \\
\hline Sociabilidade & 0,045 \\
\hline Escolaridade & 0,945 \\
\hline Total & 0,091 \\
\hline Retraimento & 0,512 \\
\hline Somático & 0,021 \\
\hline Ansiedade/depressão & 0,019 \\
\hline Contato social & 0,961 \\
\hline Pensamento & 0,691 \\
\hline Problema de atenção & 0,758 \\
\hline Delinquência & 0,909 \\
\hline Agressividade & 0,920 \\
\hline Problemas sexuais & 0,615 \\
\hline Escala introversão & 0,046 \\
\hline Escala extroversão & 0,916 \\
\hline Escala total & 0,108 \\
\hline
\end{tabular}

$\mathrm{A}=$ resposta de conteúdo animal; $\mathrm{Ad}=$ resposta com conteúdo de detalhe animal; $\mathrm{C}=$ determinante cromático (cor) sem forma; $\mathrm{CBCL}=$ Child Behavior Checklist; $\mathrm{CF}=$ determinante cromático (cor) com forma indefinida; $\mathrm{C}^{\prime}=$ determinante acromático (sem cor) sem forma; C'F = determinante acromático (sem cor) sem forma definida; $\mathrm{FC}=$ determinante cromático (cor) com forma; Fm = determinante de movimento inanimado com forma definida; $\mathrm{H}$ = resposta de conteúdo humano; $\mathrm{M}=$ determinante de movimento humano; $\mathrm{m}=$ determinante de movimento inanimado sem forma; $\mathrm{mF}$ $=$ determinante de movimento inanimado sem forma definida.
Os resultados apontam diferenças estatisticamente significantes nas variáveis do Rorschach que medem a capacidade do sujeito de controlar suas emoções, como a porcentagem de respostas com determinantes cromáticos (cor) sem forma definida $(\% \mathrm{CF})(\mathrm{p}=0,041)$, e seus impulsos, através da porcentagem de determinantes com movimentos inanimados sem forma, mais aqueles com forma indefinida $(\% \mathrm{~m}+\mathrm{mF})(\mathrm{p}=0,013)$. Ambos os resultados indicam uma dificuldade no controle de impulsos e emoções nos sujeitos que permaneceram em tratamento. Também foram encontradas diferenças nas escalas de sociabilidade $(p=0,045)$, ansiedade e depressão $(\mathrm{p}=0,019)$, somática $(\mathrm{p}=0,021)$ e introversão $(p=0,046)$ no CBCL. Nas variáveis medidas nos testes Bender e WISC III, não foram encontradas diferenças estatisticamente significantes, assim como nas demais variáveis do Rorschach e do CBCL.

\section{Discussão}

As pesquisas de abandono de psicoterapia de crianças realizadas até o momento possibilitaram uma melhor definição do abandono prematuro, a identificação de fatores que levam ao abandono, bem como de formas de prevenir e melhorar os serviços psicoterápicos oferecidos às crianças ${ }^{3,4}$. Entretanto, a investigação na área é ainda escassa, e esta limitação parece estar relacionada com a dificuldade de acesso aos pacientes que abandonam prematuramente o tratamento, já que estes raramente se dispõem a participar de pesquisas longitudinais e acabam por limitar o entendimento dinâmico do tema.

$\operatorname{Kazdin}^{11}$ salienta a importância de caracterizar os moderadores que influenciam os resultados de uma intervenção para o encaminhamento dos pacientes ao tratamento mais efetivo de acordo com suas necessidades. Neste estudo em particular, a análise dos resultados encontrados na comparação entre o G1 e o G2 demonstra que a criança que permaneceu em tratamento era predominantemente do sexo feminino, apresentava sintomas de ansiedade, insegurança, medos, depressão, queixas somáticas e de dificuldades na sociabilidade no momento da busca - todos estes sintomas característicos dos transtornos internalizantes - e recebeu atendimento com uma frequência de duas vezes por semana. Além disso, as crianças do G1 apresentaram diferenças estatisticamente significantes em algumas das variáveis medidas pelo Rorschach, indicando uma maior dificuldade de controlar os impulsos e emoções do que o G2.

Estes resultados demonstram que as crianças que possuem estas características sociodemográficas (sexo feminino), clínicas (transtornos internalizantes e maior frequência de atendimentos) e nos resultados dos testes são aquelas que têm uma maior chance de permanecerem em psicoterapia psicanalítica, beneficiarem-se com o tratamento e não abandonarem prematuramente o mesmo. O G2, por sua vez, foi caracterizado por um número acentuado de meninos que 
apresentavam problemas de comportamento e falta de atenção no momento da busca - estes predominantemente comportamentos externalizantes - que receberam atendimento uma vez por semana ou que só realizaram a avaliação antes de interromperem o tratamento. Esses resultados indicam que as crianças que possuem as características descritas acima têm mais chance de não se beneficiarem com a psicoterapia psicanalítica e de interromperem o tratamento em sua fase inicial.

Os resultados deste estudo também confirmam o alto índice de abandono encontrado nas psicoterapias com crianças ${ }^{5}$. É relevante salientar, entretanto, que índices elevados de abandono são também encontrados nas psicoterapias em diferentes idades, grupos diagnósticos e modalidades de tratamento (30-60\%), denotando que este não é um problema restrito à população infantil que realiza psicoterapia psicanalítica. Estudos realizados no Brasil sobre o tema encontraram índices de abandono que variam de 35 a $68,7 \%$ nos centros que oferecem serviços psicológicos ${ }^{12-14}$. Recente amostra pesquisada em Porto Alegre (RS) sobre a efetividade da psicoterapia psicanalítica com adultos reportou taxas de desistência ainda maiores, entre 76,4 e $94,12 \%{ }^{15}$. Apesar disso, $88,24 \%$ dos pacientes desta amostra descreveram sua experiência com o tratamento como satisfatória. Estes achados comprovam dados de outros informes sobre a tendência dos psicoterapeutas de subestimarem as altas e superestimarem as interrupções.

Neste estudo sobre abandono de psicoterapia com crianças, procurou-se fazer uma investigação longitudinal, através da aplicação do CBCL, com os abandonantes, mas o retorno das famílias foi pouco significativo, confirmando evidências citadas na literatura ${ }^{3}$. Acredita-se, porém, que similares resultados poderiam ser encontrados em relação à satisfação com o tratamento: apesar das desistências, o índice de satisfação seria elevado. Essa suposição se deve aos resultados encontrados em um levantamento informal realizado com os pais no ano de 2006 nesta mesma instituição: quase $100 \%$ dos pais cujos filhos estavam em tratamento no momento do levantamento se consideravam plenamente satisfeitos ou satisfeitos com a psicoterapia psicanalítica realizada com seus filhos até aquele momento (Deakin EK, Dian S, Satisfação dos pais com a psicoterapia dos filhos, trabalho não publicado, 2006).

Em estudo realizado sobre as características das crianças que abandonam o tratamento, concluiu-se que a continuidade do tratamento não depende muito das características da criança, mas sim de fatores situacionais e externos à psicoterapia, como raça e classe social ${ }^{16,17}$. Alguns fatores internos, contudo, podem ser detectados na avaliação, como, por exemplo, a motivação dos pais, e podem ser utilizados no planejamento do tratamento a ser oferecido à criança.

Algumas hipóteses levantadas sobre as razões pelas quais esta amostra apresentou um alto índice de abandono merecem ser mencionadas. Em primeiro lugar, como referido anteriormente, a psicoterapia psicanalítica parece ser mais efetiva no tratamento das crianças do sexo feminino que apresentam transtornos internalizantes. Estes resultados confirmam os achados encontrados em pesquisa realizada no Anna Freud Center, em Londres, Reino Unido ${ }^{18}$, no qual o estudo retrospectivo de 763 prontuários demonstrou que a psicanálise com crianças e modalidades oriundas desta são mais efetivas no tratamento de transtornos emocionais como a ansiedade e a depressão, não demonstrando a mesma efetividade para transtornos de conduta. Também existem evidências que apontam que são as crianças do sexo masculino aquelas que tendem ao maior abandono da psicoterapia ${ }^{19}$.

A amostra inicial deste estudo era composta predominantemente de meninos que apresentavam problemas de comportamento, portanto transtornos externalizantes. Para esta população, estudos indicam serem as psicoterapias comportamentais as mais indicadas, e o elevado índice de desistência reforça a ideia de que a psicoterapia psicanalítica não apresenta a mesma efetividade ${ }^{18,20}$.

Em segundo lugar, cabe ressaltar que os terapeutas envolvidos eram, na sua maioria, iniciantes e inexperientes quanto à realização da psicoterapia com crianças. Estudos reportam uma correlação positiva entre a quantidade de tempo de trabalho e os resultados do tratamento ${ }^{21}$. Em geral, os terapeutas iniciantes tendem a criar expectativas ideais e não reais de seu trabalho, aspirando algo perfeito e além do esperado pelo paciente ${ }^{22}$.

Não obstante, vale mencionar que a maioria dos terapeutas que trabalham a partir de uma orientação psicanalítica ou psicodinâmica, sejam estes iniciantes ou experientes, objetiva ir além do alívio dos sintomas que motivaram a busca do tratamento e visa a uma mudança estrutural da criança, na qual há o estabelecimento de uma nova relação objetal ${ }^{23}$. Para a obtenção desses objetivos, é necessária, muitas vezes, uma maior frequência das sessões e uma duração mais prolongada do tratamento. Entretanto, nem sempre os pais buscam essas mudanças, prevalecendo a normalização do comportamento da criança como o objetivo principal. Quando esta é atingida, mesmo que outros objetivos possam existir por parte do terapeuta, os pais podem se sentir beneficiados e optar por interromper o tratamento, o que, para eles, não é considerado prematuro, já que suas expectativas foram atendidas ${ }^{24}$. Além disso, é importante ressaltar que os profissionais que trabalham a partir de uma orientação psicodinâmica como a psicoterapia psicanalítica tendem a interpretar o término do tratamento como resistência ou falta de motivação por parte do paciente e/ou sua família. Estes últimos, por sua vez, tendem a atribuir o término a fatores externos à psicoterapia, como falta de tempo, dificuldades de transporte ou financeira ${ }^{25}$. Plunkett ${ }^{26}$ salienta que quando as expectativas dos pais se mostram incongruentes com as do serviço no qual a criança está inserida há uma maior chance de que a família interrompa o tratamento e/ou tenha dificuldade de participar deste. $\mathrm{O}$ autor cita algumas formas de prevenção do abandono prematuro como a compreensão dos objetivos e expectativas dos pais e da criança frente ao tratamento, bem 
como a formação de uma forte aliança terapêutica com estes, já que no caso da psicoterapia com crianças, a aliança terapêutica com os pais é mais importante do que a estabelecida com a criança ${ }^{27}$.

É importante ressaltar que, neste estudo, não foi feita uma análise inicial das expectativas dos pais, e, talvez, uma maior ênfase tenha que ser dada a esta questão em estudos futuros sobre efetividade e abandono de psicoterapia com crianças. Recente estudo ${ }^{28}$ demonstrou que a satisfação da criança/adolescente está associada a expectativas positivas do terapeuta. Já uma maior satisfação dos pais apareceu associada a um maior número de sessões e melhora no funcionamento global da criança. Cabe ressaltar, entretanto, que os constructos relativos às expectativas e satisfação parentais frente à psicoterapia ainda são vagos e necessitam ser mais bem definidos para que possam ser utilizados nos estudos de avaliação de intervenções terapêuticas com crianças.

Vale a pena lembrar aqui a posição de Gabbard \& Westen $^{29}$. Para estes autores, a teoria moderna de ação terapêutica diz respeito à utilização de estratégias (sejam estas psicanalíticas ou não) que facilitarão o processo de mudança proposto no plano de tratamento de determinado paciente. Com isto, os autores sugerem que o profissional deve se preocupar mais com o fato de uma técnica ser terapêutica para determinado paciente e não com se ela se enquadra dentro da linha teórica seguida por este mesmo profissional. Dentro desta mesma linha de pensamento, Tryon \& $\mathrm{Kane}^{27}$ propõem que não se deve oferecer o mesmo tipo de tratamento a todas as crianças independente da situação, pois ao se desconsiderarem as características individuais de cada caso, corre-se o risco de maiores índices de abandono de tratamento.

A necessidade de gratificação imediata, a pouca capacidade reflexiva das crianças e dos seus pais e a busca de soluções rápidas e de baixo custo podem também estar relacionadas a um maior índice de abandono das psicoterapias de longa duração como as propostas pela psicoterapia psicanalítica. Além disso, existe a constante pressão dos planos de saúde que querem cortar custos a qualquer preço e, por isso, limitam a qualidade do serviço a ser oferecido. A distância entre o tratamento real e o tratamento ideal para determinado paciente parece ser cada vez maior, comprometendo os resultados e levando a maiores índices de abandono. Cabe aos profissionais que atuam nesta área unir seus esforços na compreensão das demandas características da atualidade para que, assim, possam continuar oferecendo seus serviços de forma efetiva a toda a comunidade infantil que apresenta problemas psicológicos. Por fim, é fundamental a integração entre o clínico e as universidades, locais nos quais são fomentadas as pesquisas, para que, então, os importantes conceitos desenvolvidos pela psicanálise infantil ao longo do último século possam ser aplicados efetivamente ao paciente que se apresenta nos dias de hoje, prevenindo, com isto, o abandono prematuro do tratamento.

\section{Referências}

1. Sourander A, Jensen P, Davies M, Niemelä S, Elonheimo H, Ristkari T, et al. Who is at greatest risk of adverse long-term outcomes? The Finnish from the boy to a man study. J Am Acad Child Adolesc Psychiatry. 2007;46(9):1148-61.

2. Gould MS, Shaffer D, Kaplan D. The characteristics of dropouts from a child psychiatric clinic. J Am Acad Child Adolesc Psychiatry. 1985;24(3):316-28.

3. Armbruster P, Kazdin AE. Attrition in child psychotherapy. In: Ollendick TH, Prinz RJ, eds. Advances in clinical child psychology. New York: Plenum; 1994.

4. Kazdin AE. Holland L, Crowley M. Family experience of barriers to treatment and premature termination from child therapy. J Counsult Clin Psychol.1997;65(3):453-63.

5. Kazdin AE. Dropping out of child psychotherapy: issues for research and implications for practice. Clin Child Psychol Psychiatry.1996;1(1):133-56.

6. Koppitz EM. O teste gestáltico Bender para crianças. 2nd ed. Porto Alegre: Artes Médicas; 1987.

7. Figueiredo VL. Adaptação e padronização Brasileira da escala de inteligência Wechsler para crianças. 3rd ed. São Paulo: Casa do Psicólogo; 2002.

8. Kroeff P. Normas brasileiras para o teste de Bender. Psicol Reflex Crit. 1988;1/2(3):12-9.

9. Exner JE, Weiner IB. The Rorschach: A comprehensive system volume 3: assessment of children and adolescents. New York: John Wiley \& Sons Inc; 1995.

10. Achenbach TM, McConaughy SH, Howell CT. Child/adolescent behavioral and emotional problems: implications of cross-informant correlations for situational specificity. Psychol Bull. 1987;101(2):213-32.

11. Kazdin AE. Evidence-based treatment and practice: new opportunities to bridge clinical research and practice, enhance the knowledge base, and improve patient care. Am Psychol. 2008;63(3):146-59.

12. Lopez MA. Considerações sobre o atendimento fornecido por clínicas-escolas de psicologia. Arq Bras Psicol. 983;35(2):123-35.

13. Carvalho RM, Térziz A. Caracterização da população atendida na clínica-escola do Instituto de Psicologia PUCCAMP. Est Psicol. 1988;5(1):112-25.

14. Lhullier AC, Nunes ML, Antochevis AF, Porto AM, Figueiredo D. Mudança de terapeuta e abandono de psicoterapia em uma clínica-escola. Aletheia. 2000;11:7-11.

15. Jung SI, Fillippon AP, Nunes ML, Eizirik CL. Avaliação de resultados da psicoterapia psicanalítica. Rev Psiquiatr RS. 2007;29(2):184-96.

16. Viale-Val G, Rosenthal RH, Curtiss G, Marohn RC. Drop out from adolescent psychotherapy: a preliminary study. J Am Acad Child Adolesc Psychiatry. 1984;23(5):562-8.

17. Weisz JR, Weiss B, Longmeyer DB. Giving up on child psychotherapy: who drops out? J Counsult Clinl Psychol. 1987;55(6):916-8.

18. Target M, Fonagy P. The efficacy of psychoanalysis for children: prediction of outcome in a developmental context. J Am Acad Child Adolesc Psychiatry. 1994;33(8):1134-44.

19. Lasky RG, Salomone PR. Attraction to psychotherapy: influences of therapist status and therapist-patient age similarity. J Clin Psychol. 1977;33(2):511-6.

20. Kazdin AE. Methodology, design and evaluations in psychotherapy research. In: Bergin AE, Garfield SL, eds. Handbook of psychotherapy and behavior change. 4th ed. New York: John Wiley \& Sons Inc; 1994. p. 19-71.

21. Sandell R, Blomberg J, Lazar A, Carlsson J, Broberg J, Schubert J. Varieties of long-term outcome among patients in psychoanalysis and long-term psychotherapy. A review of findings in the Stockholm Outcome of Psychoanalysis and Psychotherapy Project (STOPP). Int J Psychoanal. 2000;81(Pt 5):921-42.

22. Gabbard GO. Elaboração e término. In: Gabbard GO. Psicoterapia psicodinâmica a longo prazo: texto básico. Porto Alegre: ArtMed; 2005. p. 161-78.

23. Sours J. Uma abordagem analiticamente orientada à avaliação diagnóstica. In: Glenn J, ed. Psicanálise e psicoterapia de crianças. Porto Alegre: Artes Médicas; 1996. p. 373-90.

24. Soley G, Hooper I, Marshall R, Chambliss C. Educating new therapists about differences between parents' and therapists' expectations of child psychotherapy. Resources in education. Collegeville, USA: Ursinus College; 1998. http://www.eric. ed.gov/ERICDocs/data/ericdocs2sq1/content_storage_01/0000019b/80/16/ff/ed.pdf

25. Todd DM, Deane FP, Bragdon RA. Client and therapist reasons for termination: a conceptualization and preliminary validation. J Clin Psychol. 2003;59:133-47.

26. Plunkett JW. Parents' treatment expectations and attrition from a child psychiatric service. J Clin Psychol. 1984;40:372-7.

27. Tryon G, Kane AS. The helping alliance and premature termination. Counsel Psychol Quart. 1993;3:233-8.

28. Garland AF, Haine RA, Boxmeyer CL. Determinates of youth and parent satisfaction in usual care psychotherapy.. Eval Program Plann. 2007;30(1):45-54.

29. Gabbard GO, Westen D. Rethinking therapeutic action. Int $\mathbf{J}$ Psychoanal. 2003;84(Pt 4):823-41. 